\title{
Focus on emotion and disorders of emotion
}

\author{
Regulation of emotion is becoming better understood, but despite the societal costs of disorders like depression \\ and anxiety, their causes remain unclear. Five review articles in this issue provide perspective on this literature.
}

Individual quality of life depends on our ability to experience emotions appropriately and to regulate them in response to stressful events. In addition, depression and anxiety are a substantial public health burden. For example, in the course of one year in the United States, 40 million people experience an anxiety disorder and 21 million people experience some form of depression. For these reasons, it is important to understand how the brain regulates emotions, how this regulation becomes impaired by disorders of emotion, and which therapies may be effective in treating these disorders. In this issue, a special focus containing four reviews and a perspective explores recent advances in this important field.

Fear conditioning can occur directly, by experiencing fear, or indirectly, by observing another animal experiencing fear. Elizabeth Phelps and Andreas Olsson open the focus by comparing the social learning of fear in humans and other animals, combining the neurobiology of fear learning and social neuroscience to produce a model of how social interactions could influence fear learning in the brain. They propose that social fear learning differs from direct fear learning in that the reaction of the fearful conspecific acts as the unconditioned stimulus in social learning, and that verbal threat information is likely to evoke plasticity in the hippocampus, and not the amygdala.

The remaining pieces explore the causes and treatment of anxiety and depression. These disorders have a lot in common, with related symptoms being accompanied by similar brain activity patterns in overlapping populations. Patients with an anxiety disorder have a $90 \%$ chance of being diagnosed with depression over their lifetime. Depression and anxiety respond to the same treatments, including selective serotonin reuptake inhibitors and cognitive-behavioral therapy. Rates of failure to respond to treatment are also similar. Both classes of disorder are associated with decreased hippocampal volume, probably as a result of changes in glia and neuropil rather than of neuron death. In addition, the same variant of the serotonin transporter gene has been associated with vulnerability to both anxiety and depression.

One approach to studying the pathways involved in depression is to determine which effects are shared between different antidepressant treatments. Treatments for depression, including both drugs and electroconvulsive therapy, increase the expression of brain-derived neurotrophic factor (BDNF) with a slow onset similar to the time course of their clinical effectiveness. Activity in the subgenual cingulate cortex is increased by sadness and decreased by successful antidepressant treatment and during natural recovery from depression. Sustained amygdala activation before treatment predicts responsiveness to cognitive-behavioral therapy. Many treatments for depression also stimulate neurogenesis in laboratory animals, although these findings have not been confirmed in human patients. Thus, all these processes have been suggested as possible causes of depression.
However, effectively treating depression does not necessarily illuminate its cause. In their perspective, Keri Martinowich, Husseini Manji and Bai Lu explain that deficits in BDNF signaling do not appear to lead to depression or anxiety in animal models, although decreases in hippocampal BDNF accompany stress-induced depression. In contrast, mice lacking BDNF or its receptor fail to respond to antidepressants by showing reduced immobility in the forced-swim test, which is used to screen for effective antidepressant drugs. These results suggest that BDNF may be important for antidepressant treatment without being a cause of depression. Similarly, the review from Amar Sahay and René Hen concludes that neurogenesis in the hippocampus is probably not a major contributor to the etiology of depression, but may be required for the behavioral effects of antidepressants. Prevention of neurogenesis does not produce a depression or anxiety phenotype, despite evidence that depression treatments stimulate neurogenesis in animal models.

Individual susceptibility to depression and anxiety in response to life stressors may be related to genetic variation. In their review, Klaus-Peter Lesch and Turhan Canli explore how individual variation in the serotonin transporter gene may interact with personality, emotion regulation and social cognition. This fascinating idea has been controversial because the initial association study, like most findings on the genetics of complex disorders, has been replicated inconsistently. This variability might be due to small effect sizes or may indicate that the positive results are a result of statistical artifacts. In light of the inherent difficulties with association studies for multi-factorial disorders, neuroscientists may need to provide supporting evidence by identifying mechanisms that could link transporter function with depression.

Of course, the motivation behind this research is to find new treatments for human disorders. In the final review, Kerry Ressler and Helen Mayberg examine treatments for depression that have grown out of basic neuroscience, including vagal nerve stimulation, transcranial magnetic stimulation and deep brain stimulation. Basic knowledge of the circuits involved in fear learning is now supporting research aimed at manipulating learning to treat—and even prevent—anxiety disorders. Enhancing extinction of learned fear responses as a treatment for anxiety disorders is now in clinical trials. Drugs that block fear learning after a traumatic event may help to reduce the risk of developing post-traumatic stress disorder. Because memories are consolidated not only after they are originally formed, but also after each time that they are recalled, a similar approach may prove useful for established anxiety disorders as well.

The research described in this focus has made substantial progress toward understanding the brain systems that regulate emotion and has opened up a range of practical applications. We hope that these reviews help to clarify this complicated literature, and that the focus may inspire further basic and clinical work on these important problems. 